\title{
Silencing XIAP suppresses osteosarcoma cell growth, and enhances the sensitivity of osteosarcoma cells to doxorubicin and cisplatin
}

\author{
YANG QU, PENG XIA, SHANYONG ZHANG, SU PAN and JIANWU ZHAO \\ Department of Orthopedics, The Second Hospital of Jilin University, \\ Nanguan, Changchun, Jilin 130042, P.R. China
}

Received October 10, 2014; Accepted December 3, 2014

DOI: $10.3892 /$ or.2014.3698

\begin{abstract}
X-chromosome-linked inhibitor of apoptosis protein (XIAP) is an important member of the inhibitors of apoptosis (IAP) family. It has been shown that XIAP promotes the invasion, metastasis, growth and survival of malignant cells, and confers resistance to some chemotherapeutic drugs in various types of cancer. However, little is known regarding its detailed role in osteosarcoma (OS). In the present study, we first investigated the expression of XIAP in OS tissues, and an increased expression of XIAP in OS tissues compared to adjacent non-tumor tissue was identified. Additionally, its expression level correlated with key pathological characteristics including clinical stage, tumor size and metastasis. Subsequently, small interfering RNA (siRNA) was used to block XIAP expression to evaluate the effect of XIAP siRNA on cell proliferation, colony formation, cell cycle, apoptosis, tumorigenicity, and the combined effects of doxorubicin or cisplatin in OS cell lines (MG63 cells). Downregulation of XIAP expression using the RNA silencing approach efficiently decreased cell proliferation and colony formation, and induced cell apoptosis and cell cycle in the G0/G1 stage. In addition, this downregulation inhibited tumor growth in a nude murine model. The resuls also showed that treatment with XIAPshRNA in combination with doxorubicin or cisplatin enhanced chemosensitivity. These results suggested that XIAP may aid the diagnosis of OS and may be an effective strategy for gene therapy of this disease.
\end{abstract}

\section{Introduction}

Osteosarcoma (OS) is the most common primary bone malignancy in children and adolescents $(1,2)$. Although neoadjuvant

Correspondence to: Professor Jianwu Zhao, Department of Orthopedics, The Second Hospital of Jilin University, 218 Ziqiang Street, Nanguan, Changchun, Jilin 130042, P.R. China

E-mail: zhaojiawu14208@sohu.com

Key words: osteosarcoma, XIAP, cisplatin, doxorubicin, apoptosis chemotherapy followed by surgical excision has ameliorated the long-term survival of OS patients, most patients that do not respond to commonly used drugs, such as cisplatin and doxorubicin, have a poor prognosis due to intrinsic or acquired drug resistance $(3,4)$. Identification of the critical molecules and/or signal transduction pathways responsible for regulating the development to drug resistance is therefore required to optimize therapeutic options or develop novel effective therapy for $\operatorname{OS}(5,6)$.

At the molecular level, a number of aberrations have been identified that contribute to this chemoresistance. The deregulated expression of members of the inhibitor of apoptosis protein (IAP) family, such as X-chromosome-linked inhibitor of apoptosis protein (XIAP) and survivin $(7,8)$, have been shown to influence the sensitivity towards chemotherapeutic agents. XIAP, an important member of the IAP family proteins, binds to and inhibits the activities of caspase-3, -7 or -9 , leading to apoptosis inhibition (9). XIAP is extensively expressed in many types of cancer and is not expressed or expressed at a substantially lower level in normal tissue counterparts (10-12). Accumulating evidence showed that inhibition of XIAP expression using antisense oligonucleotides or small-interfering RNA (siRNA) suppressed tumor cell proliferation and invasion, induced cell apoptosis, and suppressed tumor growth (13-18). In addition, Harlin et al reported that XIAP knockout mice are phenotypically normal with no significant pathological characteristics (19). XIAP is considered one of the most important factors involved in resistance to the apoptotic effects of drugs and radiation in tumor cells (20). It has been shown to be one of the important regulators in cisplatin- and doxorubicin-induced apoptosis in some cancer cells $(21,22)$ and downregulation of XIAP sensitizes cells to cisplatin and doxorubicin $(13,23)$. Therefore, XIAP is considered to be an attractive target with respect to the molecular therapy of cancer. However, for XIAP, the possible roles and whether increase resistance of doxorubicin and cisplatin in OS remain unclear.

The present study was undertaken to evaluate the expression and its clinical diagnostic significance in OS patients and to analyze its functional role, especially with respect to chemoresistance. Our results showed that the downregulation of XIAP expression by short hairpin RNA (shRNA) 
significantly inhibited MG63 cell proliferation and colony formation, induced cell arrest in the G0/G1 and cell apoptosis, and suppressed OS tumor growth in a nude murine model. Furthermore, downregulation of XIAP significantly enhanced the chemosensitivity of doxorubicin and cisplatin in OS cells. These experimental data suggested that XIAP was associated with the development of human OS and silencing XIAP is a potential strategy therapy for human OS.

\section{Materials and methods}

Patients and tissue samples. This study was approved by the Research Ethics Committee of the Second Hospital of Jilin University (Changchun, China). Written informed consent was obtained from all the patients.

Sixty OS patients ( 36 male and 24 female) treated in the Department of Orthopedics, The Second Hospital of Jilin University, Changchun, China, between August 2008 and August 2013, were enrolled retrospectively in this study. The human OS biopsy specimens were obtained from primary lesions. The biopsies were performed prior to chemotherapy or radiotherapy for diagnostic purposes. Clinicopathological characteristics were recorded from the subjects, such as personal information, diagnosis, disease stage, metastasis and tumor characteristics. The selection criteria were selected as clear pathological diagnosis, full medical records, and completed follow-up data. Patients were excluded when a previous or secondary malignancy was identified. Normal tissue samples adjacent to the tumor were removed $5 \mathrm{~cm}$ away from the peripheral tumor cells, and the lack of tumor cell infiltration was verified by pathological examination.

Immunohistochemistry. Samples were divided into the OS group and tissue adjacent to tumor, which was stained against XIAP, in the two treatment groups. Briefly, the sections were dewaxed in xylene, rehydrated in descending alcohols, and blocked for endogenous peroxidase and avidin/biotin activities. After blocking with $3 \%$ BSA in $0.01 \mathrm{M}$ PBS, the sections were incubated with mouse monoclonal antibody against human XIAP (Santa Cruz Biotechnology, Inc., Santa Cruz, CA, USA) at a dilution of $1: 1,000$ overnight at $4^{\circ} \mathrm{C}$. The samples incubated with PBS instead of the primary antibody were used as the negative control. The samples were then incubated with horseradish peroxidase (HRP)-labeled goat anti-rabbit secondary antibody for $2 \mathrm{~h}$ at room temperature. After washing with PBS three times, immunostaining was visualized using a streptavidin-peroxidase reaction system, and developed with diaminobenzidine (DAB) $-\mathrm{H}_{2} \mathrm{O}_{2}$ (Wuhan Boster Biological Technology Ltd., China) and then observed under an optical microscope.

Immunoreactivity was graded according to the percentage of positive tumor cells $(0,0 \% ; 1<10 \% ; 2,10-50 \% ; 3,51-80 \%$; $4,80-100 \%)$, and the intensity of staining ( 0 , no staining; 1, weak; 2, moderate; and 3, strong). These scores were calculated by multiplying the intensity score to the percentage score. The section with a final score of $<4$ being classified as negative, and a score of $\geq 4$ as positive.

shRNA design and plasmid construction. XIAP-shRNA (GenBank accession no. NM_001167) and non-specific
shRNA sequences were designed using the siRNA target design tools (Ambion Inc., Austin, TX, USA), XIAP-shRNA and non-specific shRNA were synthesized as follows: XIAP-shRNA sense: 5'-GATCCACTGGACAGAGAAAGAG CCTTCAAGAGAGGCTCTTTCTCTGTCCAGTTTTTTTG GAAA-3' and antisense: 5'-AGCTTTTCCAAAAAAACGGA CAGAGAAAGAGCCTCTCTTGAAGGCTCTTTCTCTGT CCAGTG-3'; non-specific-shRNA sense: 5'-GATCCTTCTCC GAACGTGTCACGTTCAAGAGAAAACTACTTCTTTTAC CTTAGA-3' and antisense: 5'-AGCTTCTTCTTTCTCCG AACGTGTCACGTCTCTTGAAAAACTACTTCTTTTACC TTG-3', where the sequence did not target any gene product and where there was no significant sequence similarity to human gene sequences. The oligonucleotides were annealed and then ligated into the BamHI and HindIII sites of pSilencer 6.1-CMV neo expression vector according to the manufacturer's instructions (Ambion). The two recombinant plasmids were verified by DNA sequencing. The plasmid encoding XIAP-shRNA was referred to as pSi-XIAP, and the plasmid encoding non-specific shRNA, which was used as a negative control, was designated as $\mathrm{pSi}-\mathrm{NC}$.

Cell culture and transfection. Human MG63 OS cells were obtained from the Cell Bank of the Type Culture Collection of Chinese Academy of Sciences, Shanghai Institute of Cell Biology (Shanghai, China). MG63 cells were grown in DMEM medium (HyClone, Logan, UT, USA) supplemented with $10 \%$ fetal bovine serum (FBS, Gibco, Carlsbad, CA, USA), $2 \mathrm{mM}$ glutamine, $100 \mathrm{U} / \mathrm{ml}$ penicillin and $100 \mathrm{mg} / \mathrm{ml}$ streptomycin at $37^{\circ} \mathrm{C}$ in a humidified atmosphere containing $5 \% \mathrm{CO}_{2}$.

MG63 cells were seeded in 6-well plates at $2.0 \times 10^{4}$ cells $/$ well, respectively, and cultured overnight to $80 \%$ confluence prior to transfection. The cells were then transfected with plasmid pSi-XIAP or plasmid pSi-NC using Lipofectamine ${ }^{\mathrm{TM}} 2000$ (Invitrogen, Carlsbad, CA, USA) in medium according to the manufacturer's instructions. G418 $(800 \mu \mathrm{g} / \mathrm{ml}$, Sigma, St. Louis, MO, USA) was used to screen stably transfected clones. Stable transfectants were designated to as MG63/pSi-XIAP and MG63/pSi-NC. After $48 \mathrm{~h}$, quantitative real-time polymerase chain reaction (RT-qPCR) and western blot analysis were performed to evaluate the effect of gene silencing.

Quantification by real-time polymerase chain reaction. Total RNA of OS tissue and cells was isolated using TRIzol reagent following the manufacturer's instructions (Invitrogen), and quantified with the Nanodrop 2000 (Thermo, Japan). First-strand cDNA synthesis and amplification were performed using reverse transcription reagents (Takara, China) according to the manufacturer's instructions. RT-qPCR assays were carried out using SYBR-TAQ real-time kits (Takara Biotechnology, Otsu, Japan) and amplification equipment ABI Prism 7900 HT (Applied Biosystems, Foster City, CA, USA). GADPH was used as the endogenous control for quantifying mRNA levels. Primers used for of XIAP and GAPDH were: XIAP sense: 5'-GACAGTATGCAAGATGAGTCAAGTCA-3' and antisense: 5'-GCAAAGCTTCTCCTCTTGCAG-3'; GAPDH sense: 5'-TGTGGGCATCAATGGATTTGG-3' and antisense: 5'-ACACCATGTATTCCGGGTCAAT-3'. The qPCR conditions were as follows: a pre-denaturing at $95^{\circ} \mathrm{C}$ for $5 \mathrm{~min}$, followed by 40 cycles of denaturation at $95^{\circ} \mathrm{C}$ for $10 \mathrm{sec}$, 
annealing/extension at $60^{\circ} \mathrm{C}$ for $20 \mathrm{sec}$, within final extension of $72^{\circ} \mathrm{C}$ for $5 \mathrm{~min}$. The amplification specificity was assessed by the melting curve analysis. The $2^{-\Delta \Delta C t}$ method was used to calculate the relative abundance of target gene expression generated using the Rotor-Gene 6000 Series Software 1.7 (Qiagen, Hilden, Germany).

Western blotting. After $48 \mathrm{~h}$ of transfection, MG63 cells were trypsinized, lysed in RIPA lysis buffer $(\mathrm{pH} 7.4,150 \mathrm{mM}$ $\mathrm{NaCl}, 0.1 \%$ SDS, $0.5 \%$ sodium deoxycholate, $1 \% \mathrm{NP}-40$ in PBS, protease complete inhibitor; Roche Diagnostics $\mathrm{GmbH}$, Mannheim, Germany), frozen and thawed three times, centrifuged at $13,000 \mathrm{x} \mathrm{g}$ for $20 \mathrm{~min}$ at $4^{\circ} \mathrm{C}$ to remove insoluble material, and then the supernatant was harvested as total proteins for experiments. Total protein concentration was determined using the BCA assay kit (Sigma). Cell extracts (50 $\mu \mathrm{g}$ of protein) were separated by $8-15 \%$ SDS-PAGE gel and transferred to nitrocellulose membranes. After blocking non-specific binding sites with 5\% dried milk in PBST, the membranes were incubated with anti-cleaved caspase-3 (1:1,500; Santa Cruz Biotechnology, Inc.), anti- $\beta$-actin (1:5,000; Sigma-Aldrich, St. Louis, MO, USA) anti-cleaved PARP $(1: 2,000)$ and anti-XIAP $(1: 2,000)$ (both from Santa Cruz Biotechnology, Inc.) antibody in PBST containing 3\% BSA overnight at $4^{\circ} \mathrm{C}$, followed by incubation for $2 \mathrm{~h}$ at room temperature with the appropriate HRP secondary antibody diluted $(1: 3,000)$ in PBST containing $3 \%$ BSA. Protein bands were visualized with enhanced chemiluminescence reagent (ECL; Amersham, GE Healthcare, Velizy-Villacoublay, France). Densitometry was performed by Quantity One image analysis software.

Cell proliferation and colony formation. The MTT assay was used to determine the effect of downregulated XIAP on cell proliferation. Briefly, MG63 cells transfected with pSi-XIAP and pSi-NC, along with untreated cells were seeded in 96-well plates at a density of $5 \times 10^{3}$ cells/well. At indicated time points, $20 \mu \mathrm{l}$ methyl thiazol tetrazolium (MTT) solution $(5 \mathrm{mg} / \mathrm{ml})$ was added to each well and cultured for $4 \mathrm{~h}$. Centrifugation was performed to remove the supernatant, and $200 \mu \mathrm{l}$ of DMSO was added to each well followed by agitation for $10 \mathrm{~min}$ to dissolve the crystals. Absorbance was measured at $570 \mathrm{~nm}$ with a microplate reader (Molecular Devices Corp., Sunnyvale, CA, USA), and growth inhibition was calculated. All the experiments were performed in triplicate.

For the colony formation assay, MG63 cells transfected with plasmid pSi-XIAP or pSi-NC were seeded in a 6-well plate at a low density $\left(1 \times 10^{3}\right.$ cells/well), and then cultured for 7 days. The cells were fixed with $4 \%$ paraformaldehyde for 20 min and counted after staining with $1 \%$ crystal violet. The experiments were carried out in triplicate wells at least three times.

Cell cycle analysis. MG63 cells were plated in 10-cm dishes and treated with plasmid pSi-XIAP or pSi-NC for $48 \mathrm{~h}$. The cells were trypsinized, fixed with $70 \%$ ethanol, and incubated overnight at $-20^{\circ} \mathrm{C}$. The cells were pelleted and resuspended in $200 \mu \mathrm{l}$ of PBS with $50 \mu \mathrm{l}$ DNAase-free, RNase A, and incubated at $37^{\circ} \mathrm{C}$ for $1 \mathrm{~h}$. Propidium iodide (PI, $750 \mu \mathrm{l}$ ) was added and incubated at room temperature for $15 \mathrm{~min}$. The DNA contents of samples were measured using a FACS-Calibur ${ }^{\mathrm{TM}}$ flow cytometer (BD Biosciences, San Jose, CA, USA). The flow data obtained from the samples were then analysed by CellQuest software (BD Biosciences).

Cell apoptosis. Cell apoptosis was detected using flow cytometry. Briefly, at $24 \mathrm{~h}$ after transfection, a total of $1 \times 10^{6}$ cells were digested with $10 \mu \mathrm{g} / \mathrm{ml}$ RNase for $30 \mathrm{~min}$ at $37^{\circ} \mathrm{C}$. Annexin V-fluorescein isothiocyanate $(0.5 \mu \mathrm{g} / \mathrm{ml})$ and PI $(0.6 \mu \mathrm{g} / \mathrm{ml})$ were then added to a $250 \mu \mathrm{l}$ aliquot of this cell suspension. After a 15-min incubation in the dark at room temperature, the sample was read on a Coulter Epics XL flow cytometer (Beckman Coulter Inc., Brea, CA, USA), and the percentage of apoptotic cells was calculated using CellQuest software (BD Biosciences). Experiments were performed in triplicate. In addition, cleaved caspase- 3 and cleaved PARP protein expression were determined by western blotting using specific antibody as an additional indicator of apoptosis.

In vitro drug treatment. MG63 cells transfected with plasmid pSi-XIAP or pSi-NC were cultured for $24 \mathrm{~h}$, and the medium was changed to DMEM containing indicated concentrations of doxorubicin and cisplatin. After $72 \mathrm{~h}$ of treatment, cell proliferation was assessed using an MTT assay, and cell apoptosis was measured by flow cytometry. In addition, caspase-3 activity was determined by the caspase colorimetric protease assay kits (Millipore Corporation, Billerica, MA, USA), following the manufacturer's instructions, as an additional indicator of apoptosis.

Tumor growth in vivo. To investigate the effects of downregulated XIAP by RNAi on the tumorigenicity of xenograft and the influence on survival of tumor-burdened animals, 30 female BALB/nude mice (aged 4-6 weeks) were purchased from the Jilin Institute of Experimental Animals. The study protocol was approved and mice were maintained in accordance with the Institutional Guidelines of the Experimental Animals of Jilin University.

Stable MG63/pSi-XIAP and MG63/pSi-NC clone cells and untreated MG63 cells $\left(1 \times 10^{8}\right)$, respectively, were subcutaneously injected into the right flank of mice. The animals were sacrificed on the 21st day after injection, tumor tissues were resected and the volume and weight were measured. In addition, spleen tissue was collected and cultured for a splenocyte surveillance study using the MTT assay as previously described (30).

Statistical analysis. Data are presented as mean \pm SD (standard deviation). Statistical analysis between two samples was performed using the Student's t-test. Statistical comparison of $>2$ groups was performed using one-way ANOVA followed by a Tukey post-hoc test. The relationship between XIAP expression levels and clinical and pathological variables was analysed using Pearson's Chi-square test. Graphpad Prism 5.01 software (GraphPad Software, San Diego, CA, USA) and SPSS ${ }^{\circledR} 16.0$ (SPSS Inc., Chicago, IL, USA) for Windows ${ }^{\circledR}$ were used for statistical analyses. $\mathrm{P}<0.05$ was considered to indicate a statistically significant difference.

\section{Results}

$X I A P$ is upregulated in OS patients and correlates with clinical characteristics of OS patients. To identify the potential 
A

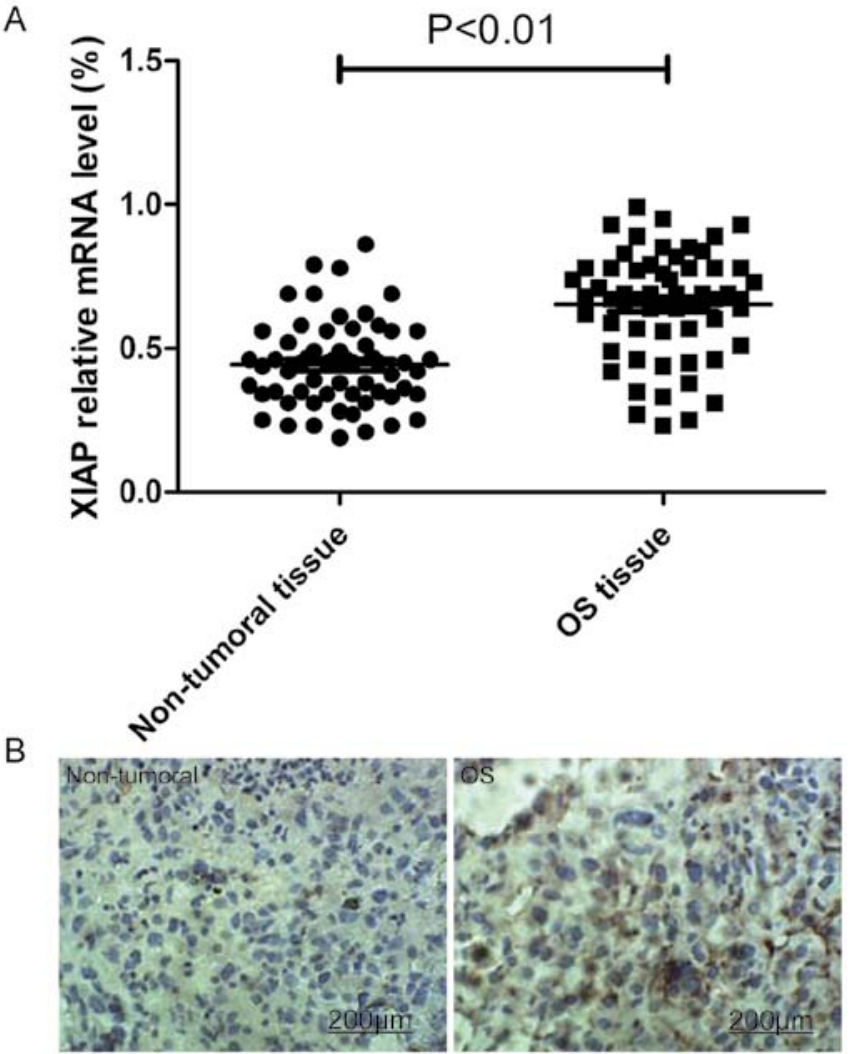

Figure 1.Levels of XIAP are increased in OS clinical samples. (A) Quantitative RT-PCR results of relative expression level of XIAP in 60 cases of OS tissue samples and adjacent non-tumoral tissue samples. The mRNA expression level of GAPDH was quantified as an internal standard and used to normalize the level of XIAP from the same sample. (B) Immunohistochemical results of XIAP expression in OS tissue samples and matched adjacent non-tumoral tissue samples, $\mathrm{P}<0.01$ vs. non-tumoral tissue.

roles of XIAP in the development and progression of OS, we detected XIAP expression levels in tumor tissues and adjacent non-tumoral tissues from 60 patients with OS by RT-qPCR and immunohistochemistry. As revealed by the RT-qPCR analysis, the mRNA expression level of XIAP was significantly upregulated in tumoral tissues compared to expression in the matched adjacent non-tumoral tíssues $(\mathrm{P}<0.05$; Fig. $1 \mathrm{~A})$. In addition, elevated levels of XIAP protein were found in OS tumoral tissues compared with the paired adjacent non-tumoral tissues from the same patients as shown by immunohistochemical staining (Fig. 1B).

The associations of XIAP protein expression with various clinicopathological parameters of OS tissues was analyzed. XIAP protein was significantly upregulated in OS patients with advanced clinical stage, larger tumour size $(>8 \mathrm{~cm})$ and positive distant metastasis as compared to those with low clinical stage, small tumor size $(<8 \mathrm{~cm})$ and without distant metastasis (all $\mathrm{P}<0.05)$ (Table I). No correlations occurred between XIAP protein levels and patient age, gender and anatomic location.

Silencing XIAP causes effective and specific downregulation of XIAP expression in MG63 cells. We constructed a plasmid (pSi-XIAP) that is capable of expressing a shRNA that targets the XIAP, and then transfected this plasmid into MG63 cells. The mRNA and protein expression intensities of XIAP were
Table I. The correlation of XIAP expression with clinicopathological characteristics of osteosarcoma.

\begin{tabular}{llll}
\hline Clinical factor & Positive & Negative & P-value \\
\hline
\end{tabular}

$\begin{array}{llll}\text { Age, years } & & & \\ <20(\mathrm{n}=39) & 30 & 9 & >0.05 \\ \geq 60(\mathrm{n}=21) & 15 & 6 & \end{array}$

Gender

$\begin{array}{llll}\text { Male }(\mathrm{n}=36) & 28 & 8 & >0.05 \\ \text { Female }(\mathrm{n}=24) & 17 & 7 & \end{array}$

Clinical stage
I-IIA $(\mathrm{n}=31)$

IIB-III ( $\mathrm{n}=29)$

Distant metastasis

Absent $(\mathrm{n}=41)$

Present $(\mathrm{n}=19)$

Anatomic location

Tibia/femur $(n=46)$

Other location $(\mathrm{n}=14)$

Tumor size, $\mathrm{cm}$

$<8(\mathrm{n}=38)$

$\geq 8(n=22)$

\section{0}

25

27

$18-1$

36

9

25

20 analyzed by RT-qPCR and western blotting which showed that plasmid pSi-XIAP markedly decreased the expression of XIAP compared with the control and pSi-NC groups (Fig. 2A and B). The above results indicated that the expression of XIAP gene in MG63 cells was downregulated specifically and effectively by plasmid pSi-XIAP.

Silencing XIAP inhibited cell proliferation and colony formation in MG63 cells. The anti-proliferative effect of XIAP silencing on MG63 tumor cells was examined using the MTT assay following MG63 cell transfectection with individual plasmid. Downregulation of XIAP by pSi-XIAP could significantly inhibited the proliferation of MG63 tumor cells compared with the control and pSi-NC groups $(\mathrm{P}<0.01 ;$ Fig. $3 \mathrm{~A})$.

The effects of silencing XIAP on tumor cell colony formation were determined in MG63 cell lines by analyzing cell colony formation at 14 days after transfection with the indicated plasmid. Cell colony formation in the pSi-XIAP groups was significantly reduced compared to the control and $\mathrm{pSi}-\mathrm{NC}$ groups ( $\mathrm{P}<0.05 ;$ Fig. 3B). No significant difference was identified between the $\mathrm{pSi}-\mathrm{NC}$ and control groups $(\mathrm{P}>0.05)$.

Silencing XIAP induces cell arrest and apoptosis in MG63 cells. To determine the effects of silencing XIAP on the cell cycle, FACScan flow cytometry assays were performed. A flow cytometric analysis revealed that G1-phase cell population was increased in the pSi-XIAP group compared to the control and pSi-NC groups ( $\mathrm{P}<0.05$; Fig. 4A and $\mathrm{B})$. In addition, silencing XIAP resulted in a much lower percentage of cells in $\mathrm{S}$ phase compared with those of the control and $\mathrm{pSi}-\mathrm{NC}$ groups $(\mathrm{P}<0.05$; Fig. 4A and $\mathrm{B})$. 
A

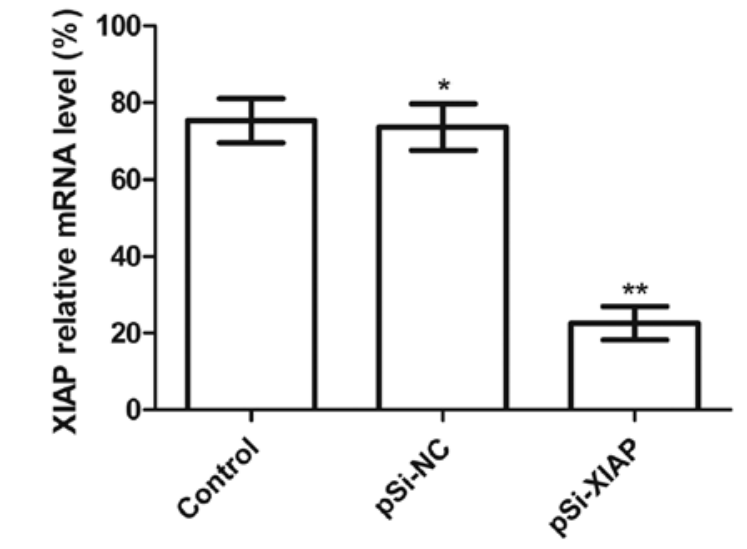

B

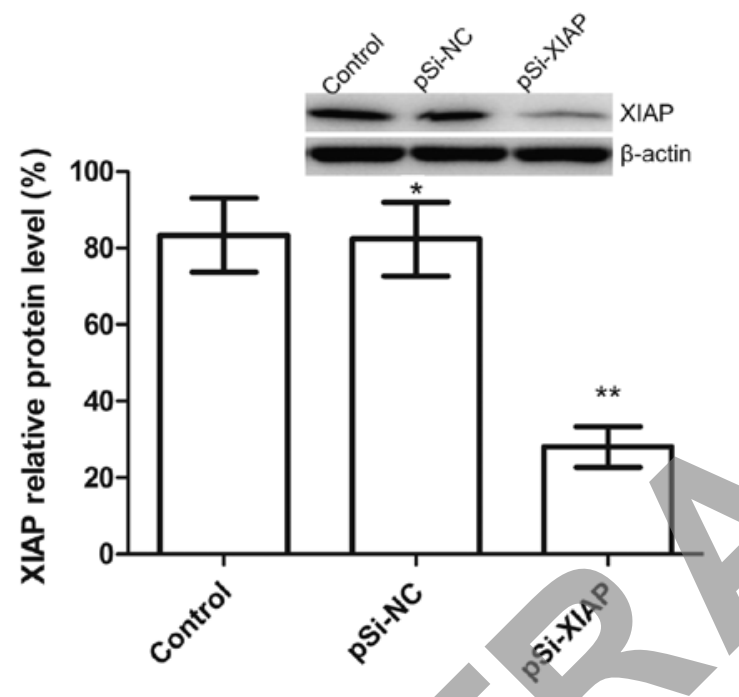

Figure 2. Silencing XIAP inhibited the XIAP expression in MG63 cells (A) Quantitative RT-PCR analysis of XIAP in MG63 cells $48 \mathrm{~h}$ after transfection with indicated plasmid. (B) Western blot analysis of XIAP in MG63 cells $48 \mathrm{~h}$ after transfection with indicated plasmid. $\beta$-actin was used as an internal control. ${ }^{*} \mathrm{P}<0.05,{ }^{* *} \mathrm{P}<0.01$ vs. control.

In order to investigate the effect of silencing XIAP on cell apoptosis in MG63 cells, flow cytometry assays were performed. Flow cytometric analysis showed that cells transfected with plasmid pSi-XIAP significantly induced cell apoptosis compared to untreated cells and cells transfected with plasmid pSi-NC ( $\mathrm{P}<0.05$; Fig. 4C).

To determine the potential mechanism of cell apoptosis in vitro, cleaved PARP and cleaved caspase- 3 protein expression were detected using western blotting. It was found that cleaved caspase- 3 and cleaved PARP protein expression was significantly increased in pSi-XIAP treatment groups compared to the control and pSi-NC groups (Fig. 4D).

Downregulation of XIAP sensitizes OS cells to doxorubicin and cisplatin. To investigate whether downregulation of XIAP expression has the potential to sensitize OS cells to chemotherapy, a combination treatment of XIAP-specific siRNA with anticancer drugs was performed. Twenty-four hours after transfection with plasmid pSi-XIAP, cells were treated with doxorubicin and cisplatin at indicated concentrations for $72 \mathrm{~h}$, respectively. To evaluate the growth inhibitory effects
A
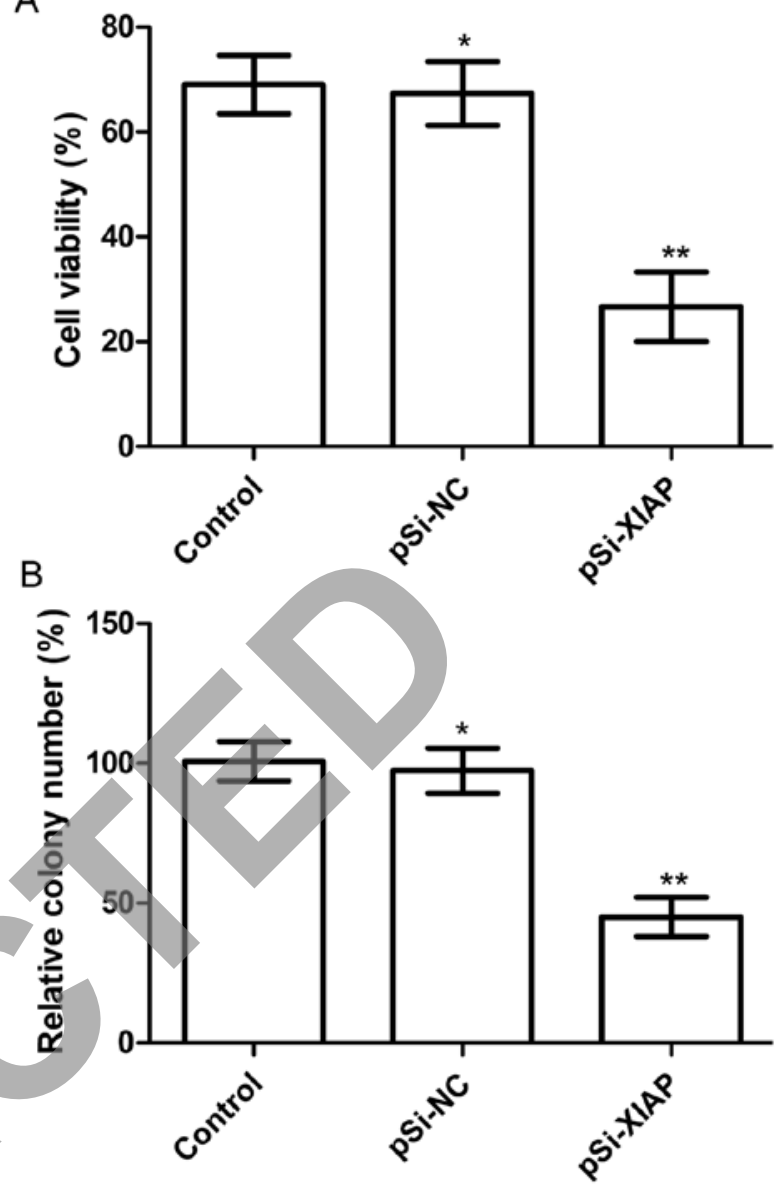

Figure 3. Silencing XIAP inhibited cell proliferation and colony formation in MG63 cells. (A) Cell viability was determined by the MTT assay in MG63 cells transfected with the indicated plasmids. (B) Cell colony formation was detected in MG63 cells transfected with indicated plasmids. All the assays were performed in triplicate. ${ }^{*} \mathrm{P}<0.05,{ }^{* *} \mathrm{P}<0.01$ vs. control.

of pSi-XIAP in combination with doxorubicin or cisplatin in MG63 cells, MTT assays were performed. As shown in Fig. 5A, pSi-XIAP treatment significantly enhanced the growth inhibitory effect of doxorubicin and cisplatin in MG63 cells. The pSi-NC had either no effect or only a minimal effect.

We also evaluated the effect of pSi-XIAP combination with doxorubicin or cisplatin on cell apoptosis in MG63 cells. It was found that $\mathrm{pSi}$-XIAP combination with doxorubicin or cisplatin significantly increased cell apoptosis compared to monotherapy or combination with pSi-NC $(\mathrm{P}<0.05$; Fig. 5B). In addition, we evaluated the effect of pSi-XIAP combination with doxorubicin or cisplatin on caspase-3 activity, and found that pSi-XIAP combination with doxorubicin or cisplatin could increase caspase- 3 activity compared to single drug treatment or combination with $\mathrm{pSi}-\mathrm{NC}(\mathrm{P}<0.05$; Fig. $5 \mathrm{C})$. These results suggested that downregulation of XIAP sensitizes OS cells to doxorubicin and cisplatin.

Silencing XIAP suppressed tumor growth in vivo. We also investigated the effect of silencing XIAP on tumor growth in nude mice. Tumor growth was monitored for 21 days. On day 21 , the animals were sacrificed and tumor weight and volume were measured. Our results showed that tumor weight and volume of mice treated with pSi-XIAP were significantly 
A

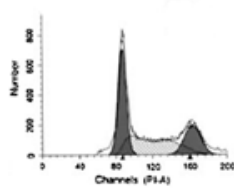

Control

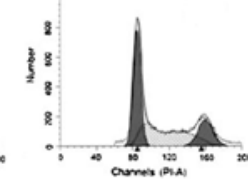

pSi-NC

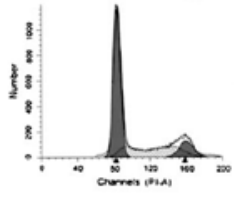

pSi-XIAP
C

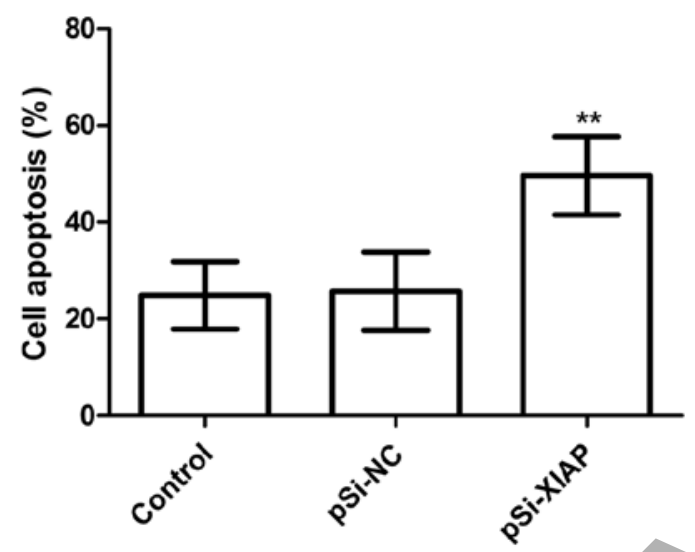

B

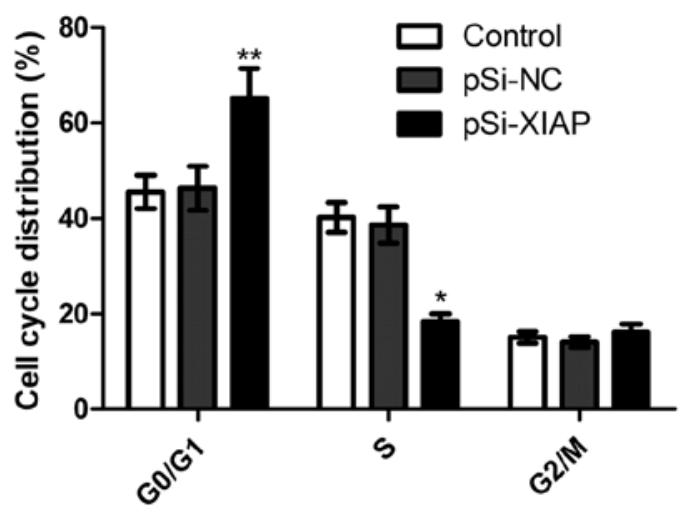

Figure 4. Silencing XIAP induced cell arrest and apoptosis in MG63 cells. (A) Cell DNA content distribution in each phase. (B) Percentage of cells distributed in each phase of the cell cycle. (C) Cell apoptosis was detected in MG63 cells transfected with indicated plasmids. (D) Western blot analysis of cleaved PARP and cleaved caspase-3 in MG63 cells after transfection with indicated plasmid. $\beta$-actin was used as an internal control. " $\mathrm{P}<0.05$, ${ }^{* *} \mathrm{P}<0.01$ vs. control.

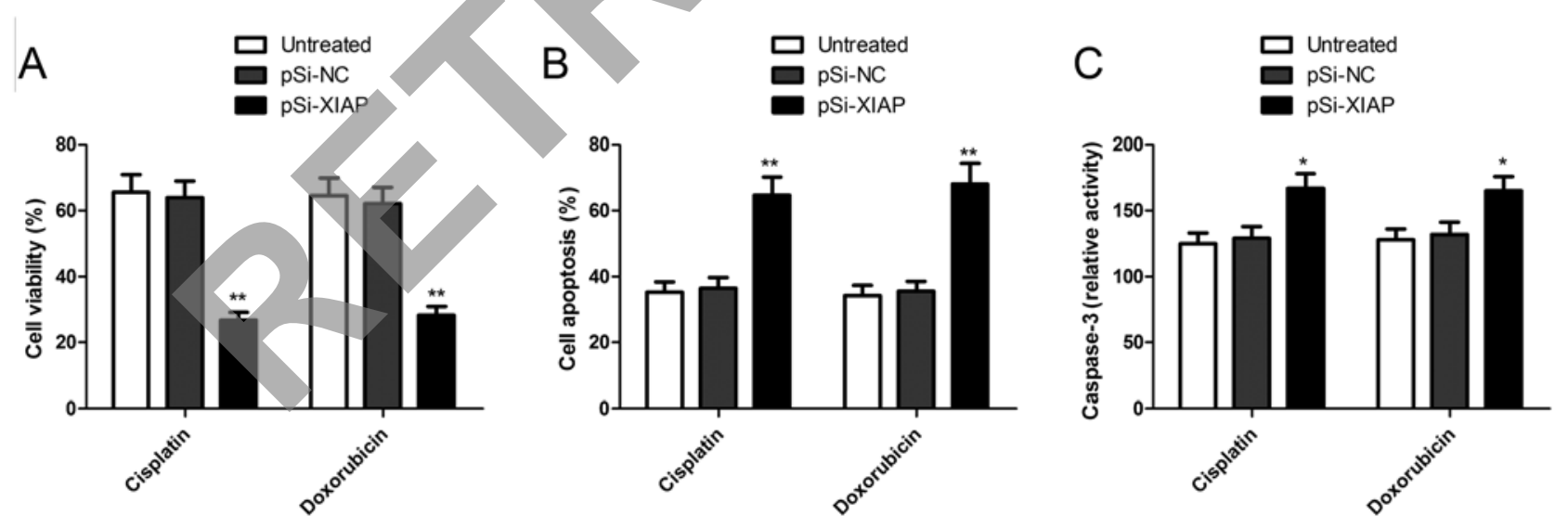

Figure 5. Effects on cell proliferation and apoptosis of plasmid pSi-XIAP in combination with doxorubicin or cisplatin in MG63 cells. MG63 cells were transfected with plasmid pSi-XIAP or pSi-NC for $24 \mathrm{~h}$, and then treated with indicated concentrations of doxorubicin or cisplatin. After $72 \mathrm{~h}$, cell proliferation was determined by (A) MTT assay, apoptosis was analyzed by (B) flow cytometry, and (C) caspase- 3 activity was determined by caspases colorimetric protease assay kits. ${ }^{*} \mathrm{P}<0.05,{ }^{* *} \mathrm{P}<0.01$ vs. plasmid pSi-NC combination with doxorubicin or cisplatin.

reduced when compared to the control and $\mathrm{pSi}-\mathrm{NC}$ groups $(\mathrm{P}<0.05$; Fig. 6A-C). We employed MTT assay in modulating splenocyte proliferation to demonstrate the antitumor activities. We found that splenocyte cell proliferation of pSi-XIAP group was significantly decreased compared to the control and pSi-NC groups $(\mathrm{P}<0.01$; Fig. 6D). These results indicated that suppression of XIAP expression in OS cells markedly inhibited their tumorigenicity in nude mice.

\section{Discussion}

It is well known that inhibition of apoptosis contributes to tumorigenesis by aberrantly prolonging cell life span, permitting resistance to immune-based cytotoxicity, and allowing diobeyance of cell cycle check points that usually induce apoptosis (24). Several critical genes in the regulation of apoptosis have been identified, such as the anti-apoptotic 
A

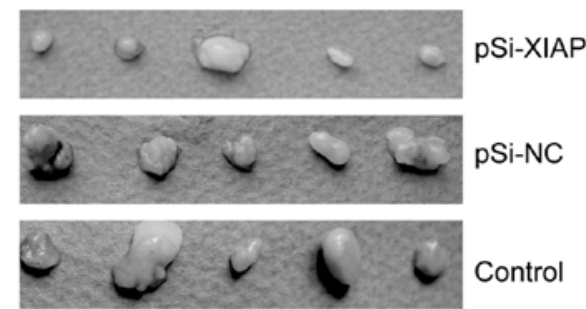

C

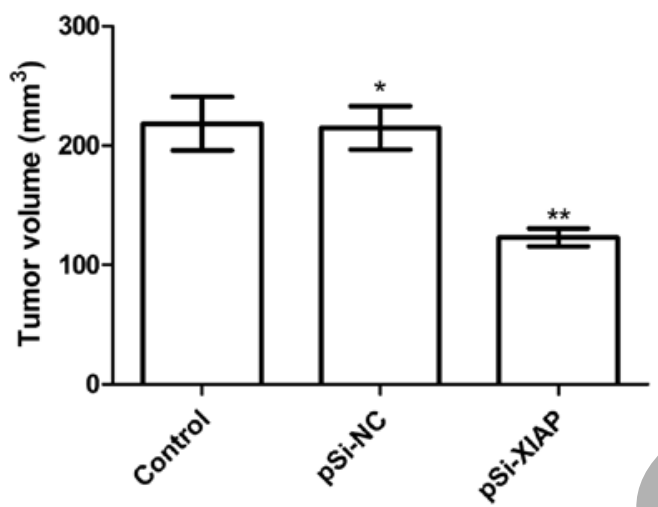

$\mathrm{B}$
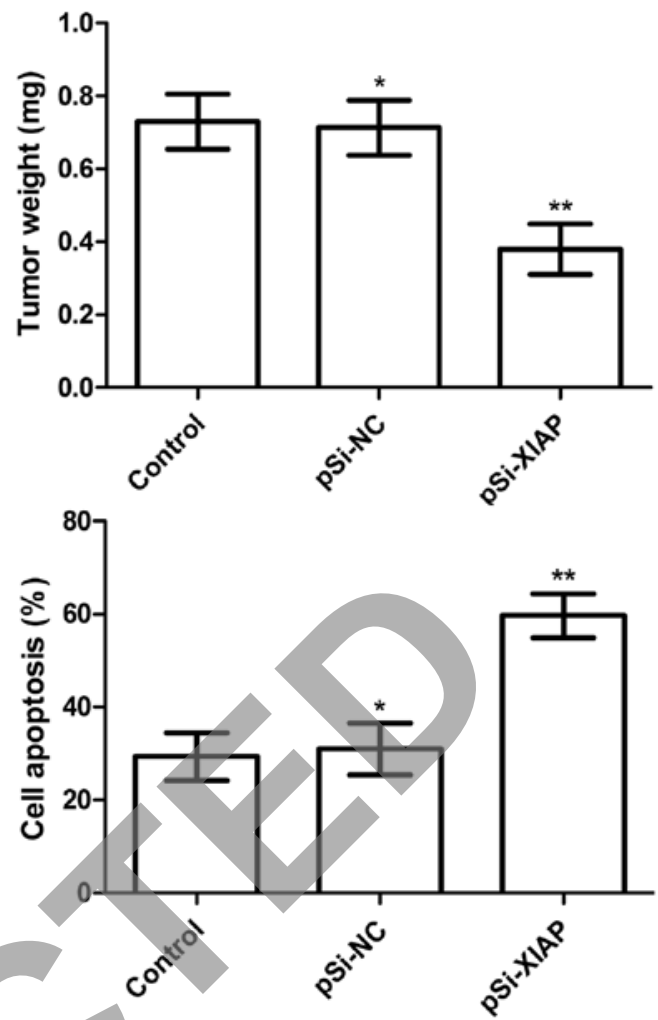

Figure 6. Silencing XIAP significantly suppressed tumor growth in nude murine model. (A) Images of tumor tissue with different plasmid treatments collected after sacrifice at day 21. (B) Tumor wet weights were measured after sacrifice at day 21. (C) Tumor volume was measured after sacrifice at day 21. (D) MTT assay of cell proliferation of splenocytes from mice in different treatment groups. ${ }^{*} \mathrm{P}<0.05,{ }^{* *} \mathrm{P}<0.01$ vs. control.

gene family IAPs and Bcl-2 family $(9,25)$. The IAPs have been identified as acting downstream of $\mathrm{Bcl}-2$ by inhibiting caspases (25-27). Eight members of IAPs have been identified in humans, and XIAP is the most potent one (28). A larger number of studies in various types of cancer cells have suggested that the expression of XIAP is elevated in various cancers and that it is important in oncogenesis $(10-12,29)$. It has been shown that the downregulated expression of XIAP inhibited cell proliferation and induced the apoptosis of many types of tumor, and sensitized cancer cells to chemotherapeutic drugs (13-24). However, the expression and detailed role of XIAP remains to be thoroughly investigated in OS. Therefore, this study mainly focuses on XIAP expression and its detailed role in OS. We first found that XIAP was elevated in most OS tissue compared to adjacent non-tumoral tissue, and its expression level correlated with key pathological characteristics, including clinical stage and metastasis. In addition, our findings show that downregulation of XIAP results in the inhibition of proliferation of MG63 cells in vitro, and suppression of solid tumor growth in a nude murine model. These results provide evidence that XIAP is required for tumor growth and that it is important in tumorigenicity for OS.

Cisplatin (cis-diamminedichloroplatinum or cisDDP/CDDP) is an anticancer drug widely used in the treatment of various types of cancer such as ovarian cancer, bladder, cervical, head and neck, esophageal and small cell lung cancer (SCLC) and OS $(13,21,23,30)$. Cisplatin-based treatment has been successful in treating some patients with OS to either prolong survival or ameliorate symptoms $(30,31)$. Other
OS patients remain inherently resistant to cisplatin, which limits the therapeutic efficacy of cisplatin (32). It has been shown that the overexpression of XIAP by adenoviral sense XIAP complementary DNA attenuated the ability of cisplatin to induce apoptosis (33). Extensive studies have shown that downregulation of XIAP using adenoviral antisense XIAP infection or siRNA increased sensitivity to cisplatin in resistant various cancer cells $(13,34,35)$. Consistent with those results, our results first showed that downregulation of XIAP by plasmid $\mathrm{pSi}$-XIAP increased sensitivity to cisplatin in resistant OS cancer cells. These findings support that XIAP is an adjuvant gene therapy target to the chemotherapy of OS.

Doxorubicin, an anthracycline antibiotic, was isolated from Streptomyces peucetius which has been widely used for the treatment of various types of cancer including lymphomas, leukemias, lung, breast, OS and ovarian cancers (36). In fact, doxorubicin is the most widely used anticancer drug with FDA approval (37). However, studies have shown that some cancer cells, including OS, are resistant to the apoptotic effects of doxorubicin (38). It has been shown that downregulation of XIAP by antisense oligonucleotide (ASO) induced cell apoptosis and increased sensitivity to doxorubicin in resistant OS cells (23). Consistent with that result, our study showed that treatment with XIAP-shRNA in combination with doxorubicin significantly induced cell apoptosis and increased caspase-3 activity, and enhanced sensitivity to doxorubicin in resistant OS cancer cells.

In conclusion, to the best of our knowledge, this is the first full-scale report concerning the association of XIAP with OS. The present study has demonstrated that XIAP was elevated in most OS patients and its expression level was correlated with 
key pathological characteristics, including clinical stage and metastasis. Additionally, downregulation of XIAP suppressed tumor growth in vitro and in vivo and increased sensitivity of OS cells to doxorubicin and cisplatin. Based on the multiple functions of XIAP in OS tumor growth, it may be considered a diagnostic marker and a potential anticancer therapeutic target for OS.

\section{References}

1. Ottaviani G, Robert RS, Huh WW, Palla S and Jaffe N: Sociooccupational and physical outcomes more than 20 years after the diagnosis of osteosarcoma in children and adolescents: limb salvage versus amputation. Cancer 119: 3727-3736, 2013.

2. Ottaviani G and Jaffe N: The epidemiology of osteosarcoma. Cancer Treat Res 152: 3-13, 2009.

3. Wittig JC, Bickels J, Priebat D, et al: Osteosarcoma: a multidisciplinary approach to diagnosis and treatment. Am Fam Physician 65: 1123-1132, 2002.

4. Meyers PA, Schwartz CL, Krailo M, et al: Osteosarcoma: a randomized, prospective trial of the addition of ifosfamide and/ or muramyl tripeptide to cisplatin, doxorubicin, and high-dose methotrexate. J Clin Oncol 23: 2004-2011, 2005

5. Yang J and Zhang W: New molecular insights into osteosarcoma targeted therapy. Curr Opin Oncol 25: 398-406, 2013.

6. Zhao Z, Tao L, Shen C, Liu B, Yang Z and Tao H: Silencing of Barkor/ATG14 sensitizes osteosarcoma cells to cisplatin-induced apoptosis. Int J Mol Med 33: 271-276, 2014.

7. Mobahat M, Narendran A and Riabowol K: Survivin as a preferential target for cancer therapy. Int J Mol Sci 15: 2494-2516, 2014

8. Wang S, Bai L, Lu J, Liu L, Yang CY and Sun H: Targeting inhibitors of apoptosis proteins (IAPs) for new breast cance therapeutics. J Mammary Gland Biol Neoplasia 17: 217-228, 2012

9. Salvesen GS and Duckett CS: IAP proteins: blocking the road to death's door. Nat Rev Mol Cell Biol 3: 401-410, 2002.

10. Devi GR: XIAP as target for therapeutic apoptosis in prostate cancer. Drug News Perspect 17: 127-134, 2004

11. Stennicke HR, Ryan CA and Salvesen GS: Reprieval from execution: the molecular basis of caspase inhibition. Trends Biochem Sci 27: 94-101, 2002.

12. Holcik M, Gibson H and Korneluk RG: XIAP: apoptotic brake and promising therapeutic target. Apoptosis 6: 253-261, 2001.

13. Ma JJ, Chen BL and Xin XY: XIAP gene downregulation by small interfering RNA inhibits proliferation, induces apoptosis, and reverses the cisplatin resistance of ovarian carcinoma. Eur J Obstet Gynecol Reprod Biol 146: 222-226, 2009.

14. Yamaguchi Y, Shiraki K, Fuke H, et al: Targeting of X-linked inhibitor of apoptosis protein or survivin by short interfering RNAs sensitize hepatoma cells to TNF-related apoptosis-inducing ligand- and chemotherapeutic agent-induced cell death. Oncol Rep 14: 1311-1316, 2005.

15. Jiang C, Yi XP, Shen H and Li YX: Targeting X-linked inhibitor of apoptosis protein inhibits pancreatic cancer cell growth through p-Akt depletion. World J Gastroenterol 18: 2956-2965, 2012.

16. Kwatra SG: Targeting x-linked inhibitor of apoptosis protein for melanoma therapy: the need for more homogeneous samples and the importance of cell lines. J Invest Dermatol 131: 797, 2011.

17. Hiscutt EL, Hill DS, Martin S, et al: Targeting X-linked inhibitor of apoptosis protein to increase the efficacy of endoplasmic reticulum stress-induced apoptosis for melanoma therapy. $\mathrm{J}$ Invest Dermatol 130: 2250-2258, 2010.
18. Mastrangelo E, Cossu F, Milani M, et al: Targeting the X-linked inhibitor of apoptosis protein through 4-substituted azabicyclo[5.3.0]alkane smac mimetics. Structure, activity, and recognition principles. J Mol Biol 384: 673-689, 2008.

19. Harlin H, Reffey SB, Duckett CS, Lindsten T and Thompson CB: Characterization of XIAP-deficient mice. Mol Cell Biol 21: 3604-3608, 2001

20. Deveraux QL and Reed JC: IAP family proteins - suppressors of apoptosis. Genes Dev 13: 239-252, 1999.

21. Cheng JQ, Jiang X, Fraser M, et al: Role of X-linked inhibitor of apoptosis protein in chemoresistance in ovarian cancer: possible involvement of the phosphoinositide-3 kinase/Akt pathway. Drug Resist Updat 5: 131-146, 2002.

22. Gagnon V, Van Themsche C, Turner S, Leblanc V and Asselin E: Akt and XIAP regulate the sensitivity of human uterine cancer cells to cisplatin, doxorubicin and taxol. Apoptosis 13: 259-271, 2008.

23. Holt SV, Brookes KE, Dive C and Makin GW: Down-regulation of XIAP by AEG35156 in paediatric tumour cells induces apoptosis and sensitises cells to cytotoxic agents. Oncol Rep 25: 1177-1181, 2011.

24. Zhang Y, Wang Y, Gao W, et al: Transfer of siRNA against XIAP induces apoptosis and reduces tumor cells growth potential in human breast cancer in vitro and in vivo. Breast Cancer Res Treat 96: 267-277,2006.

25. Oberoi-Khanuja TK, Murali A and Rajalingam K: IAPs on the move: role of inhibitors of apoptosis proteins in cell migration. Cell Death Dis 4: e784,2013.

26. Hunter AM, LaCasse EC and Korneluk RG: The inhibitors of apoptosis (IAPs) as cancer targets. Apoptosis 12: 1543-1568, 2007.

. Berthelet J and Dubrez L: Regulation of apoptosis by inhibitors of apoptosis (IAPs). Cells 2: 163-187, 2013.

28. Schimmer AD: Inhibitor of apoptosis proteins: translating basic knowledge into clinical practice. Cancer Res 64: 7183-7190, 2004.

29. Carter BZ, Kornblau SM, Tsao T, et al: Caspase-independent cell death in AML: caspase inhibition in vitro with pan-caspase inhibitors or in vivo by XIAP or Survivin does not affect cell survival or prognosis. Blood 102: 4179-4186, 2003.

30. Abe S, Nishimoto Y, Isu K, Ishii T, Goto T and Japanese Musculoskeletal Oncology G: Preoperative cisplatin for initial treatment of limb osteosarcoma: its local effect and impact on prognosis. Cancer Chemother Pharmacol 50: 320-324, 2002.

31. Anninga JK, Gelderblom H, Fiocco M, et al: Chemotherapeutic adjuvant treatment for osteosarcoma: where do we stand? Eur J Cancer 47: 2431-2445, 2011.

32. Chou AJ and Gorlick R: Chemotherapy resistance in osteosarcoma: current challenges and future directions. Expert Rev Anticancer Ther 6: 1075-1085, 2006.

33. Li J, Feng Q, Kim JM, et al: Human ovarian cancer and cisplatin resistance: possible role of inhibitor of apoptosis proteins. Endocrinology 142: 370-380, 2001.

34. Miyamoto M, Takano M, Iwaya K, et al: X-chromosome-linked inhibitor of apoptosis as a key factor for chemoresistance in clear cell carcinoma of the ovary. Br J Cancer 110: 2881-2886, 2014.

35. Cheng YJ, Jiang HS, Hsu SL, et al: XIAP-mediated protection of H460 lung cancer cells against cisplatin. Eur J Pharmacol 627: 75-84, 2010.

36. Weiss RB: The anthracyclines: will we ever find a better doxorubicin? Semin Oncol 19: 670-686, 1992.

37. Carvalho C, Santos RX, Cardoso S, et al: Doxorubicin: the good, the bad and the ugly effect. Curr Med Chem 16: 3267-3285, 2009.

38. Nobuto H, Sugita T, Kubo T, et al: Evaluation of systemic chemotherapy with magnetic liposomal doxorubicin and a dipole external electromagnet. Int J Cancer 109: 627-635, 2004. 